\title{
Research on large-scale Adhoc routing protocols
}

\author{
Wang Zhaohui and An Shuzhao \\ Qingdao Hotel Management College, Qingdao Hotel Management College \\ wangzhaohui2010@126.com,anshuzhao@126.com
}

\begin{abstract}
Based on Passive Clustering protocol in this paper, a new type of suitable for large-scale wireless sensor network routing protocol, called RBPC protocol, which USES the Passive Clustering routing Reply Broadcast routing protocol, the purpose is to make full use of the routing Reply (the Route Reply, RREP) group, the RREP Reply when routing requests in Broadcast way, a lot of $\mathrm{CH}$ nodes by receiving the grouping and routing are cached in the local, thereby reducing the Route setup times throughout the network, and reduces the source node due to establish routing packet delay.
\end{abstract}

Keywords: MIMO; Adhoc Networks; Routing

\section{Introduction}

In recent years, wireless Sensor network (WirelessAd Hoc Sensor Networks, WSN) technology have been applied in many fields, such as a wide range of environmental monitoring, intrusion detection, and disaster monitoring and management, etc. In these applications, such as in environmental monitoring, through tiny sensor deployment in the target area, you can easily obtain the environment temperature, pressure, humidity, and chemical reaction activity parameters, such as information [1]. Due to the large target monitoring area and scope of sensor node monitoring and wireless transmission range is limited, in many applications of wireless sensor nodes in the scene the battery cannot be replaced, energy is limited, and the need to deploy a huge number of [2], at the same time, due to the large number of deployment of wireless sensors will also bring a series of problems, such as the increase of the collision probability, redundancy of data transmission, and a lot of flooding overhead, etc. These problems has resulted in a serious energy waste in large-scale sensor networks, resulting in a decline in the whole sensor network life cycle.

In order to solve the problems arising from the large-scale wireless sensor networks, especially with the increase of network scale, the excessive energy consumption problems of the network clustering method has gradually been adopted, it is the effective means to expand the network range. Compared with no clumps and the mechanism of clustering can significantly improve energy efficiency, reduce the channel competition and packet collisions, and ultimately improve the network throughput. Investigate its fundamental, clustering is the network is divided into two levels, Cluster heads (Cluster Head, CH) nodes and Gateway nodes (Gateway, GW) constitute a high-level, responsible for the data sent from the ordinary node group aggregation and forwarded to the network convergence node, the sink node; Other nodes of the lower, the collection of data sent to the $\mathrm{CH}$ node.

There are many since clustering mechanism is put forward based on the network clustering routing protocol. In recent years, more and more enhanced clustering protocol is put forward, mainly focus on energy efficiency and the network life span, 
such agreements such as literature [3-4], these protocols are basically built by optimizing the election of cluster method and $\mathrm{CH}$ nodes mechanism to reduce clumps and rebuild and extend the life of the network. However, the big points cluster algorithm is prior to the events in the area is complete the establishment of the clusters, and periodically to replace $\mathrm{CH}$ nodes, as a result, a large number of control group. $\mathrm{M}$. Gerla et al., proposed in [5, 6] Passive Clustering (Passive Clustering, PC), the concept of Clustering is on demand in the network. Before is different from the traditional clustering protocol, PC through attach specific protocol information in the packet to send, don't need a cycle of explicit or signaling protocol control package, thus reducing the routing setup time and protocol overhead, and expanded the scope of network to a great extent. After many scholars did a lot in the passive clustering on the basis of research and improvement. As in document [7] the author DD (Directed coursing together [8]) and PC protocol proposed PCDD (Passive Clustering Directed coursing together) protocol, it is mainly used for interest group in the process of the broadcast in WSN, limit the participation of ordinary nodes forwarding, reduced the grouping of redundant transmission, effectively improve the performance of network. In the literature [9] the author a. Rangaswamy et al., points out that the PC protocol is faced with several problems, such as PC under low load or irregular business clusters due to control information by packet transport is not stable, under A certain network space configuration due to some nodes in the network isolation loss of critical path, some nodes when packets to not receive the correct order will lead to the destruction of the cluster or interrupt, etc. And through for PC add four new features, namely, to improve the stability of the Cluster, prevent loss of critical path, limit unnecessary broadcast, using serial number synchronization packet arrival sequence, etc., proposes PCBRP opt (Passive Cluster -based Routing Protocol, enhanced) agreement in order to solve these problems. In the literature [10] the author ECPC is presented according to the characteristics of the sensor network limited Energy (Energy Conserving Passive Clustering) agreement, the agreement according to the residual Energy of nodes and the choice of parameters such as distance better node as the cluster head nodes and gateway nodes, by reducing the overlap of the gateway node and quantity to reduce the Energy loss, and the agreement between the members of the cluster implementation cycle of sleep and work mechanism, to prolong the life cycle of the network. However, relative to the PC, this agreement will bring additional overhead in cluster formation and maintain, but in terms of performance is better than that of PC. The author in view of the network node in the document [11] business focus and node energy consumption imbalance causes network differentiation problem, put forward a new cluster head and gateway node selection of enhanced PC protocol. In the document [12] the author proposed a new algorithm ILBH (I - Inerval - Load - BalancingHeuristics), the algorithm is not need any strict conditions (such as clock synchronization, global information, etc.) and exchange of energy information between nodes will not generate additional control characteristics of the business, and the algorithm is combined with PC, put forward a new energy efficient passive clustering protocols. More and more applications based on PC and improvement can be references [12]. Based on PC protocol above improvement focuses on energy efficiency, and no further expanded its network method is put forward. We think that the main factors of restrict network scale is due to flooding in flat network broadcasting groups set up routing and produce a large amount of control overhead, though the PCDD agreement show greater advantage in reducing cost, but PCDD agreement is mainly used in data-centric network, and the main concern in this paper centered on nodes. But PCDD agreement is mainly used in 
data-centric network, and the main concern in this chapter centered on nodes of wireless sensor network (WSN); In [12] of the author in the final to AODV (Ad hoc On Demand - short Vector Routing) combined with PC AODV/PC protocol is put forward, the agreement by detecting the MAC layer, set up cluster structure, the RREQ can effectively control the size of the broadcast. Compared with AODV protocol, AODV/PC in routing load, throughput and network time delay and so on various performance indicators show greater advantage, however, AODV/PC for major Ad Hoc network applications, in the network cluster stages take into consideration of the dynamic scene, and some corresponding processing mechanism, such as dynamic refresh $\mathrm{CH}$ list, when applied in WSN, AODV/PC in the process of static scene network show some deficiencies, such as the control packet overhead significantly increased.

\section{System Models}

M. Gerla PC is put forward in the $[13,14]$ the purpose of clustering protocol is to reduce routing redundancy in the process of radio set up and the large amount of control overhead, and eventually the Ad Hoc network routing mechanism is applied to large and dense sensor networks. The agreement will sensor node is divided into key nodes (i.e., $\mathrm{CH}$ and $\mathrm{GW}$ ) and non-critical node, and only allows the key nodes in the routing request forward, eventually reduce a large amount of overhead.

The agreement on demand for network clustering, to obtain network topology, and the agreement without explicit group, all the clustering status information attached to the sent packet head, effectively reduce a large amount of control overhead.

In PC protocol, each node can have one of the five external state, namely, INITIAL CLUSTERHEAD, ORDINARY, GATEWAY and DISTRIBUTED_GATEWAY five state, in which each state represents the role of each node in the cluster structure. In the PC protocol also includes two kinds of internal state: CLUSTERHEAD_READY and GATEWAY_READY, internal state represents the state of each node tentative. In any external state of node once received packets can enter a kind of internal state, however, in a state of any internal node when sending packet will go into a kind of external state, and each node in the first time in the INITIAL state. PC protocol in the process of building cluster structure is put forward two innovation mechanism: "first declaration wins (FDW)" and "gateway selection heuristic (GSH)", FDW mechanism based on the idea of competition, the first statement of the CLUSTERHEAD node in its communication range can dominate the other nodes, become a cluster head nodes; GSH mechanism is used to determine the need to maintain connectivity between the clusters of the least GATEWAY node number, therefore, can be determined in a separate cluster in at least twoA GATEWAY node. It is worth noting that the PC protocol in the process of building cluster structure, the cluster head nodes and gateway nodes are the main participants, the use of a simple and rapid random selection strategy to determine the cluster head nodes and gateway nodes. And the agreement of grouping format did a little adjustment, in each packet MAC frame head increase two bits of a domain, used to store the current state of the node, and the receiving node by checking the domain know the sender's state information. 


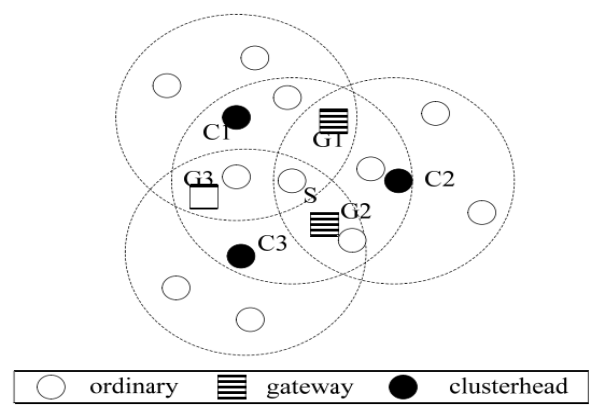

Figure 1. The PC the Clustering Structure of the Agreement

PC protocol on demand for network clustering means only when the network has a sensor nodes to send data, network clustering began, traditional clustering protocol in network before running to the node is complete before sending data, and by comparing the agreement whether on-demand features can help to reduce the system overhead. The operation of the PC protocol can use Figure 1 shows that, at first, all nodes are in the INITIAL state, when the source node $S$ there

Data send and need to build reach the sink node routing, source node $S$ routing Request (the Route Request, RREQ) packet broadcast, the adjacent nodes around the received after the group the status of the information carried by checking the frame head and realize $S$ node in the INITIAL state, the adjacent nodes and hope to forward the packet according to the condition can enter the CLUSTERHEAD, node by delaying after a period of time the packet broadcast, and insert the CLUSTERHEAD status in the group, group once successful transmission, sending node is within the scope of analysis of the $\mathrm{CH}$ nodes exist, thus into ORDINARY state, $\mathrm{C} 1, \mathrm{C} 2$ and $\mathrm{C} 3$ node in figure 1 for forwarding the packet delay time is shorter and success, and success into CLUSTERHEAD state, by detecting network business to collect their neighbors information. The G1, G2 and G3 nodes due to listen to.

The existence of multiple cluster head nodes and become a GATEWAY node, the node become ORDINARY nodes according to the PC protocol, only in the INITIAL state of the node can be a $\mathrm{CH}$ candidate nodes, and after a time no active, all nodes will fall back to the INITIAL state. As shown, these nodes and form three clusters of cluster structure. Among them, the CLUSTERHEAD nodes and GATEWAY nodes can forward broadcast information, while ORDINARY nodes do not forward broadcast packets, that can limit the number of forward broadcasts information effectively. And ORDINARY nodes and GATEWAY nodes to save their neighbor cluster header information cluster ID and used to hold information (the timestamp) the determination of the freshness of the corresponding list. If cluster of packets received from the cluster head nodes can use the list of these comparative data packet sending node ID, and add or update accordingly.

\section{Protocol improved Routing Algorithm}

The network model to make the following assumptions:

1. In a network of sensor nodes in the stationary state;

2. There are multiple sink nodes are used to collect data from the sensor node grouping, and the same as sensor nodes randomly deployed; 
3. All sensor nodes have the same capacity (CPU processing power and communication range, etc.) and have the same importance, but the sink node 4 . With unlimited energy supply and the CPU processing power;

5. The node is deployed without human management;

6. There is an optimal estimate distance based on the received signal strength mechanism available.

After the protocol in wireless Ad Hoc network research found that most of the time response packet via unicast routing is passed to the source node, the purpose is to allow only demand which received by the source node, thereby reducing the don't need these routing overhead resulted from the node to receive, because most one-on-one communication in wireless Ad Hoc networks, the probability of two nodes communicate with the same node is small, so even if an intermediate node cache reaches the destination node routing, owing to the mobile features which will be expired, thus wasted energy of the grouping and overhead. With wireless Ad Hoc networks, sink in the WSN node number is less, the node with a big probability together with the other nodes to send data to the same sink node, thus put forward the RREP packet sent in the form of radio source node, thus the RREP reply centered in a certain area of sensor node has received the packet, and cache which, by reducing The Times for the routing of the entire network set up.

The beginning of the $\mathrm{S} 1$ node has data needs to be sent to the sink node routing is available, but not so S1 radio sending RREQ packet to create routing, finally received in jump range by the sink node, and then construct the sink node a RREP packet and broadcast to all of its adjacent nodes, the RREP packet hop limit to 1 , because only the source node within a jump. Received a node RREP packet will arrive in the local cache a sink node routing, and will be discarded RREP packet will not be forwarded. Source node after receiving RREP all cached data will can send it to the sink node, when node D1 then send data routing cache before it can be used directly, and without any delay. For a period of time after S2 nodes need to establish a routing, reach the sink node grouping of RREQ when S2 send to R1 nodes, the R1 already reach the sink node routing, and construct a RREP packet and turn it in two jump range radio, but when the RREP packet radio transmission in the direction of the approximation of the sink node, because the intermediate node cache have more optimal routing, and the direction of RREP packet will be the intermediate node block, reduce the broadcast overhead. Finally within two jump and D2 S2 node receive RREP and corresponding record route at the same time, which then D2 nodes send information without waiting for the routing time delay. In order to reduce the right by the request of repeated response, each node to the maximum number of jump record once their reply RREP, in figure 5.3 reply S1 sink node routing request, reply it in the local records of RREP maximum hop count to 1, the same reply S2 R1 node requests after record the value of 2 . The purpose of this method is to reduce the number of broadcast, when R1 node after reply to the request of S2 default within two jump range all nodes will receive RREP packet, when R1 again received a RREQ packet from the other nodes, if the RREQ experience the hop count of less than 2, the $\mathrm{R} 1$ not reply response, unless the source node sending RREQ outside the range 2 to jump. At the same time, every time reply response, a routing node will refresh the RREP biggest jump value of local reply, but in order to adapt to the node network topology changes, caused by energy out every once in a while all the nodes will be the value to set to 0 . Given the nature of the RREP radio we will broadcast the scope and limit the RREP periodic method named extension ring mechanism of zero.

PC protocol of forward broadcasts a shortcoming in the node grouping random delay before a period of time, while time selection will result in the randomness of overlap between clusters and larger clusters. Node R after receiving from the $\mathrm{CH} 1$ node RREQ packet, $\mathrm{R}$ from 
the INITIAL state into the ORDINARY state, if the node can be forwarded and delayed for a short time, the first $\mathrm{R}$ node forwards the packet, as in the INITIAL state of $\mathrm{CH} 2$ node receives the packet, if also put off for a short time, the $\mathrm{CH} 2$ after successful statement CLUSTERHEAD CH1's $\mathrm{CH} 2$ clusters and cluster overlapping range is larger, so will cause the entire network nodes need to $\mathrm{CH}$ more or $\mathrm{GW}$, because these key node routing group and is responsible for the data packet forwarding, if these nodes will inevitably cause more increase of network overhead, not conducive to the expansion of the scale.

We retreat from time randomly selected in this agreement, to is determined by distance, the distance refers to the node and jump on the distance between the nodes to forward the packet, according to FreeSpace model, available:

$$
d=\frac{\lambda}{4 \pi} \sqrt{\frac{P_{t} G_{t} G_{r}}{P_{r} L}}
$$

One short to send and receive the distance between nodes, the unit is $\mathrm{m}$; Power $P_{t}$, for sending nodes for the node of the received power $P_{r}$, sending and receiving antenna gain $G_{t}$ and $G_{r}$, respectively, for electromagnetic wave wavelength $\lambda$, and the loss factor $L$ for has nothing to do with the transmission system.

Computation delay time:

$$
P=\frac{F(R-d)}{\text { CHnum }+1}
$$

Refers to the node type, on the R launch (40 m) used in the simulation, the scope of $\mathrm{d}$ for this node distance estimation algorithm are used to get the distance with a jump node, CHnum is within the scope of the node receiving the node number of $\mathrm{CH}$ nodes detected, $\mathrm{F}$ for regulating factor, to generate appropriate delay time and the value of variables associated with the cluster status of the current node, we choose the variable minimum values, when node for GW node for at ORDINARY times, when the node as the INITIAL maximum, the purpose is to make GW node as far as possible short delay time, make full use of existing GW and limit the number of nodes increases, thereby helping to reduce overhead. The choice of the variable is associated with simulation platform, the value selection starts to cause shock, in particular, node A and B neighbours, and at the same time receive A jump sending RREQ packet, when the $\mathrm{F}$ value through the hours, almost the same time delay, $\mathrm{A}$ and $\mathrm{B}$ are almost at the same time declared the CLUSTERHEAD state and received each other forward group, in accordance with the rules of the first declaration win, received another $\mathrm{CH} \mathrm{CH}$ node should I give up the current state of the group, so A and B at the same time to give up the current state and the next sends A condition statement carried in A group, when A and B received each other give up statement, respectively and the process of selecting the $\mathrm{CH}$ entered the competition, so on volatility. So, after many attempt in our simulation, we have to choose the appropriate value.

Type (5-2) means that the farther the distance jump this node, and the more $\mathrm{CH}$ nodes detected, that is, the nodes in the cross area of multiple clusters, the shorter the delay forwarding this node, and thus help to declare success.

Another noteworthy is without considering the energy efficiency of PC protocol, in order to prolong the network lifetime, we allow the node adaptive adjustment according to actual condition to send power. Whenever received a packet time point distance estimation algorithm is used to estimate the distance to the sending node and its record in the local, when the node needs to send data, if unicast data, then according to the distance from the receiver to the appropriate Settings to send power to make it just arrived at the receiver, if the radio, it is 
set to full power. In addition, because the key nodes in setting up the forward data grouping and routing to consume more energy, and best to run out of energy, in order to prolong the network lifetime, provisions in this agreement as the key node down to about $20 \%$ of the initial value, the residual energy of the node statement to exit the current state, competitive election $\mathrm{CH}$ nodes or $\mathrm{GW}$ by other node.

\section{Simulation Results}

By comparing the simulation RBPC protocol and AODV/PC in expanding network scale in terms of performance, evaluation index including control group (overhead) quantity, routing, establishing time, overlapping clusters and cluster, energy consumption, etc. AODV/PC protocol takes into consideration the dynamic scene, however, there are some local information or has the characteristics of dynamic refresh node status information, such as a node in a little time no activity will return to the INITIAL state, the node locally saved every entry in the list of adjacent nodes has a certain lifetime, $\mathrm{CH}$ list with survival in order to prevent the same $\mathrm{CH}$ nodes due to mobile inaccessible; And we will be the local information in the RBPC set to refresh, passive node even if long time no activities are still maintain the current state of adjacent nodes and $\mathrm{CH}$ list without lifetime limitation, list the entries to remove completely according to the node due to run out of energy or other reasons make a declaration of the state of the help prolong network lifetime. Special attention is, in order to compare the fairness, will more than AODV/PC protocol dynamically refresh the characteristics of the modified as same as the RBPC agreement, the performance curve of marked as "AODV/PC +". Simulation platform based on MIRAI - SF, [150], the platform is made up of NICT (National Institute of Informationand Communications Technology) development. MAC protocol using the IEEE 802.11 [17], the launch of each node distance of $40 \mathrm{~m}$, basic data rate for 11 MBPS, PLCP data rate is 1 MBPS. Sensor nodes deployed at a constant density, the number increasing from 100 to 500. Data business with Constant data Rate (Constant Bit Rate, CBR), data Rate is 1 KBPS, randomly selected from $10 \%$ of sensor nodes as a data source node, packet length 50 bytes, business lasts for $3 \mathrm{~s}$, the simulation of $300 \mathrm{~s}$.

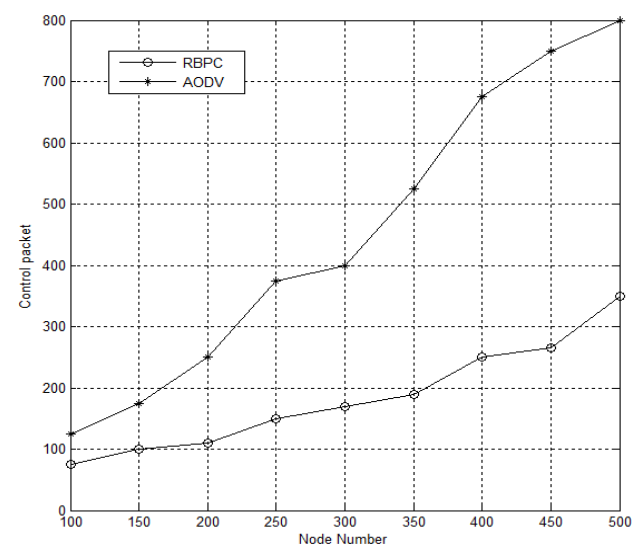

\section{Figure 2. Control Group Performance}

Shown in Figure 2 for the RBPC with AODV/PC in the control network overhead differences, we can find that compared with AODV RBPC agreement/PC can save up to $60 \%$ of control overhead, and the control overhead RBPC deals growth speed is more slow, thanks to the path 
established by extending the RBPC agreement ring way RREP radio, through radio RREP lot of potential data source routing nodes need not again started to build, and AODV/PC protocol basic node sends data needs to be set up routing, so AODV/PC would need to consume more control overhead. At the same time, the RREP broadcast when the hop limit to stop the group from flooding the entire network, so as to avoid the overhead caused by radio.

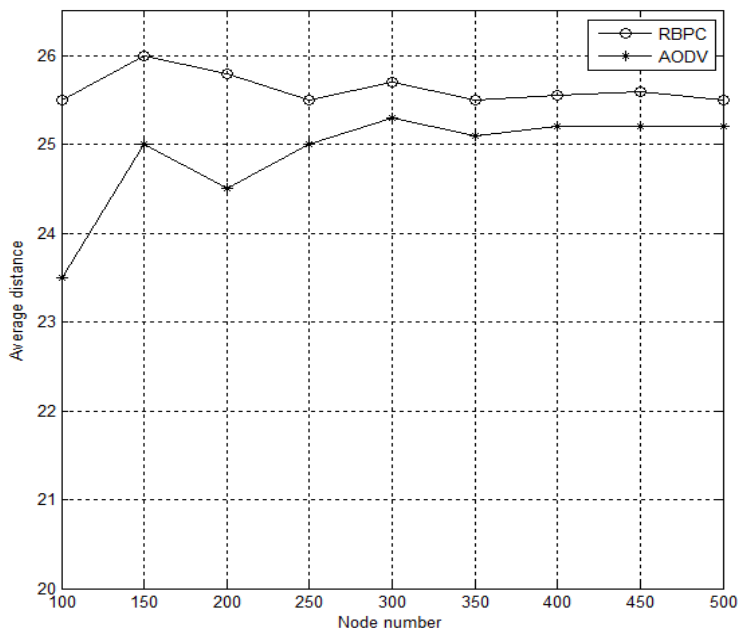

Figure 3. Overlapping Clusters and Cluster Scope

Figure 3 ordinate for GW node to the average distance of each $\mathrm{CH}$ node, the value reflects the overlap between clusters and cluster size, compared with AODV/PC, shown in the figure RBPC $\mathrm{GW}$ in the node and the average distance of the $\mathrm{CH}$ nodes is increased by $4 \%$, thereby AODV/PC protocol compared with clusters of overlapping RBPC larger clusters, largely because of AODV/PC protocol nodes in forwarding group time delay randomly selected, and the time delay in the RBPC agreement and jump on the current node to node distance and listening to $\mathrm{CH}$ node number of the current node, in general the farther distance jump node is shorter delay time, listening to the more $\mathrm{CH}$ node number has a bigger statement priority, thus reduced overlap between clusters and cluster.

\section{Conclusion}

The paper proposed the use of a passive clustering based routing reply radio RBPC routing protocol, the protocol in the PC to the network clustering on the basis of the established through radio RREP packet routing, so that a large number of potential data source node need not start routing build process, reduce the number of which is within the scope of the whole network routing, and eventually reduce the overall cost control. RREP extension ring used in radio technology limits the RREP packet in the network within the scope of the flood, avoiding the broadcast overhead caused by the growth.

\section{References}

[1] M. Gerla, T. J. Kwon and G. Pei, "On Demand Routing in Large Ad HocWireless Networks with Passive Clustering”, IEEE Wireless Communications and Networking Conference, Chicago, IL, United States, (2000) September 23-28, pp. 100-105.

[2] T. J. Kwon, M. Gerla, V. K. Varma, M. Barton and T. R. Hsing, "EfficientFlooding with Passive ClusteringAn Overhead-Free Selective Forward Mechanism forAd Hoc/Sensor Networks", Proceedings of the IEEE, vol. 91, no. 8, (2003) August, pp. 1210-1220. 
[3] V. Handziski, A. Kopke, H. Karl, C. Frank and W. Drytkiewicz, "Improving theEnergy Efficiency of Directed Diffusion Using Passive Clustering”, Wireless SensorNetworks, vol. 2920, (2004) January, pp. 172187.

[4] C. Intanagonwiwat, R. Govindan, D. Estrin and J. Heidemann, "DirectedDiffusion for Wireless Sensor Networking”, IEEE/ACM Transactions on Networking, vol. 11, no. 1, (2003) February.

[5] A. Rangaswamy and H. K. Pung, "Enhancement of passive cluster based routing protocol for mobile ad hoc networks", Computer Communications and Networks, (2002), pp. 376-381.

[6] H. Zeghilet, "Performance Improvement of Passive Clustering Algorithm inWireless Sensor Networks", 4th International Conference on Networked SensingSystems, Braunschweig, Germany, (2007) June 6-8, pp. 5356.

[7] A. S. Hafid, F. Chender and T. Jin Kwon, "Energy Aware Passive Clustering inWireless Moblie Networks", 2008 International Wireless Communications and MobileComputing Conference Conference, (2008), pp. 535-40.

[8] T. Minh Pham, T. Chi Ngo and H. Oh, "A Group Dynamic SourceRouting protocol (GDSR) using the passive clustering for wireless mobile ad hocnetworks", 2012 The 11th Annual Mediterranean Ad Hoc Networking Workshop(Med-Hoc-Net), (2012), pp. 47-54.

[9] Z. Chunhua and Tao Cheng, "A K-hop passive cluster based routing protocolfor MANET", Proceedings of the 2009 5th International Conference on WirelessCommunications, Networking and Mobile Computing (WiCOM), (2009), pp. 4.

[10] S.-S. Wang and Y.-S. Lin, "PassCAR: A passive clustering aided routingprotocol for vehicular ad hoc networks", Computer Communications, (2012).

[11] S.-S. Wang and Y.-S. Lin, "Performance Evaluation of Passive ClusteringBased Techniques for Inter-vehicle Communications", 2010 19th Annual Wireless andOptical Communications Conference (WOCC 2010), (2010) October, pp. 5.

[12] J. Luo and J. P. Hubaux, "Joint mobility and routing for lifetime elongation in wireless sensornetworks", Proc. IEEE Infocom 2005, Miami, USA, vol. 3, (2005) March 13-17, pp. 1735-1746.

[13] P. Juang, H. Oki, Y. Wang, M. Martonosi, L. S, Peh and D. Rubenstein, "Energy-efficientComputing for Wildlife Tracking Design Tradeoffs and Early Experiences With ZebraNet", Proceedings of the 10th international conference on Architectural support for programming languages and operating systems, vol. 37, no. 10, (2002) October, pp. 96-107.

[14] R. C. Shah, S. Roy, S. Jain and W. Brunette, "Data MULEs: Modeling a Three-tierArchitecture for Sparse Sensor Networks", Sensor Network Protocols and Applications, 2003. Proceedings of the First IEEE, (2003).

[15] R. S. Jain, W. Brunette, G. Borriello and S. Roy, "Exploiting Mobility for Energy EfficientData Collection in Wireless Sensor Networks", Mobile Networks and Applications, vol. 11, no. 3, (2006) June, pp. 327-339.

\section{Authors}

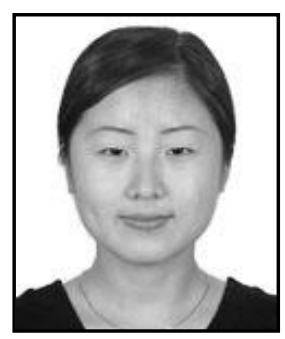

Zhaohui Wang received the Bachelor degree in Computer Engineering from Qingdao Technological University and Master's degree in Computer Engineering from China Ocean University,CHINA in 2004 and 2008 respectively. She is teaching and researching Routing Protocols at Qingdao Hotel Management College currently.

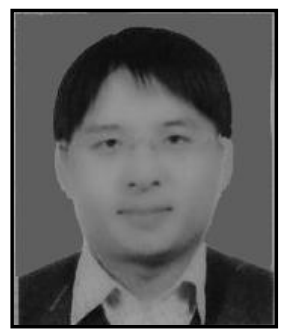

Shuzhao An received the Bachelor Degree in Science from Qufu Normal University and a master's degree in China Ocean University in 1999 and 2008 respectively.He is a $\mathrm{He}$ is a network course teachers in Qingdao Hismile College. He do some research on the network technology. 
International Journal of Grid and Distributed Computing Vol.7, No.1 (2014) 